\title{
Ag fractals formed on top of a porous $\mathrm{TiO}_{2}$ thin film
}

J. Borges ${ }^{1}$, M.S. Rodrigues ${ }^{1}$, C. Lopes $^{1}$, D. Costa $^{1}$, A. Ferreira ${ }^{1}$, R.M.S. Pereira ${ }^{2}$, M.F.

$$
\text { Costa }^{1} \text {, M.I. Vasilevskiy }{ }^{1}, \text { F. Vaz }{ }^{1}
$$

${ }^{1}$ Centro de Física, Universidade do Minho, Campus de Gualtar, 4710 - 057 Braga, Portugal

${ }^{2}$ Centro de Matemática, Centro de Física e Departamento de Matemática e Aplicações, Universidade do Minho, Campus de Gualtar, 4710-057 Braga, Portugal

\section{Abstract}

This work demonstrates the formation of $\mathrm{Ag}$ fractals on top of a $\mathrm{Ag}: \mathrm{TiO}_{2}$ thin film. The dendrite-type objects emerged from a homogeneous and highly transparent $\mathrm{Ag}: \mathrm{TiO}_{2}$ nanocomposite, via the mechanism of diffusion-limited-aggregation of $\mathrm{Ag}$ atoms, during heat-treatment at $500{ }^{\circ} \mathrm{C}$. A porous $\mathrm{TiO}_{2}$ matrix was also formed during this process, opening a wide range of possible applications, namely in sensing-based ones, together with surface enhanced spectroscopies. Furthermore, fractals incorporate a wide range of shapes and spatial scales, inducing a potentially interesting optical response, over the whole visible range, presumably related with localized surface plasmon modes with very broad spectral distribution.
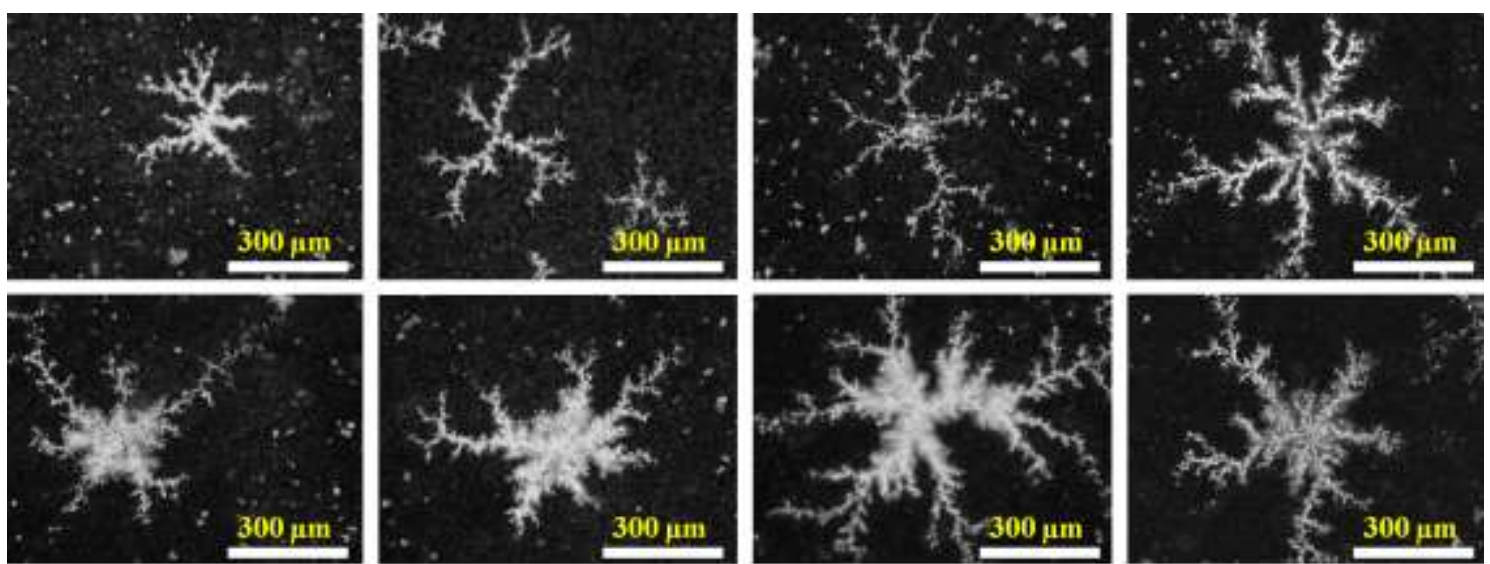

$\mathrm{Ag}$ fractals on porous $\mathrm{TiO}_{2}$ matrix, emerged from a sputtered $\mathrm{Ag}: \mathrm{TiO}_{2}$ nanocomposite thin film, via diffusion-limited aggregation (DLA) mechanism, after annealing at $500{ }^{\circ} \mathrm{C}$ 
Keywords: Silver fractals; $\mathrm{TiO}_{2}$; Surface Plasmon Resonance; Diffusion-Limited Aggregation. 


\section{Manuscript}

Plasmonic nanoparticles (NPs) such as silver (Ag) and gold ( $\mathrm{Au}$ ) can be tailored to present tuneable localized surface plasmon resonance (LSPR) bands in the visible, nearinfrared or near-ultraviolet range [1-3]. Historically, they were firstly used (via empirical trials) to control the colour of ancient Roman cups and stained glass of medieval churches. More recently, plasmonic technology has been applied in a wide range of fields, ranging from photochromic applications [4] to chemical/bio- sensors [5]. Noble nanoparticles also yield local field enhancement effects, and hence they are being used in several emerging technologies, such as magnetic storage devices [6], LEDs [7], polymer solar cells [8]; as well as in Surface Enhanced Raman Scattering (SERS), which might be used in the detection of a large variety of biomolecules [9]. More recently, it has been demonstrated that noble nanoparticles can also be used to control the emission of luminescent quantum dots (QDs) and resonant energy transfer between two QDs or a QD and a molecule, which can be enhanced when the dots interact with either propagating or localized surface plasmon polaritons [10]. Therefore, the combination of plasmonic materials with QDs is of considerable interest, both for biosensing and light-harvesting applications [11]. Furthermore, plasmonic nanoparticles can enhance significantly the photocatalytic activity of $\mathrm{TiO}_{2}$, through a mechanism mediated by the localized surface plasmons [12-14]. This ability may find important applications in the future use of solar energy, namely in the production of hydrogen by water splitting, where $\mathrm{Ag}: \mathrm{TiO}_{2}$ systems are currently being tested [15].

Despite the stunning plasmonic properties of nanocomposite materials containing noble NPs dispersed in a dielectric matrix, the width of the optical absorption due to LSPR is limited to a relatively narrow frequency range, depending on the size and distribution of the NPs, which are approximately spherical in shape $[1,2,16]$. One possible approach 
consists in elaborating NPs of complex shape, e.g. gold "nanostars"[17]. On the other hand, it has been suggested by several authors that if one can produce a fractal aggregate of nearly spherical metallic particles, the absorption spectrum broadens and can extend over a wide range of frequencies [18-23]. In fact, it has been found that inducing fractal-type aggregation in thin films can be a perspective technological tool for controlling the optical response of thin films containing plasmonic NPs [24, 25].

The present work reports the formation of Ag fractal structures, formed on top of a previously deposited $\mathrm{Ag}: \mathrm{TiO}_{2}$ nanocomposite thin film, during its thermal annealing. The dendrite-type structures are formed via diffusion-limited aggregation of Ag atoms, which are supplied to the surface via outward diffusion from the nanocomposite film, when the annealing temperature is raised up to $500{ }^{\circ} \mathrm{C}$. These aggregates show good crystal structure and a potentially interesting optical response, spanning the whole visible range, presumably related with surface plasmon modes with a very broad spectral distribution owing to their fractal geometry.

The preparation of the thin films was carried out in two steps, where the first corresponded to their preparation by reactive DC magnetron sputtering, without external heating [26, 27]. A titanium target $\left(200 \times 100 \times 6 \mathrm{~mm}^{3}, 99.8 \%\right.$ purity $)$, containing $\mathrm{Ag}$ "pellets" placed on its preferred erosion zone, was sputtered by applying a current density of 100 A.m ${ }^{-2}$, in an $\mathrm{Ar} / \mathrm{O}_{2}$ (partial pressure ratio: 7/1) plasma atmosphere (working pressure: $4.5 \times 10^{-1} \mathrm{~Pa}$ ), during $90 \mathrm{~min}$. In a second step, aiming the tailoring of the structural and morphological features of the plasmonic nanostructures, the deposited films were subjected to in-air annealing treatments, between 200 and $800^{\circ} \mathrm{C}$. The lowest annealing temperature that allowed to obtain the most well defined fractal structures was found to be $500{ }^{\circ} \mathrm{C}$. 
The atomic concentration of the as-deposited sample was measured by Rutherford Backscattering Spectroscopy (RBS) [28], showing a value for the Ag content of about 22 at. \%, and a roughly stoichiometric content for the oxide dielectric matrix $\left(\mathrm{TiO}_{2}\right)$. The optical transmittance and reflectance spectra were measured between 250 and $2500 \mathrm{~nm}$, before and after the annealing treatments of the films. Since the films were deposited on single crystal Si (100) substrates, it was only possible to obtain transmittance spectra for wavelengths above $1200 \mathrm{~nm}$, where Si is semi-transparent.

According to Fig. 1, there are clearly major changes when the films are subjected to the annealing treatments, as revealed by the particular shape of the reflectance and transmittance curves. The interference fringes, observed in the reflectance spectra of the as-deposited sample, almost disappear after the annealing at $500{ }^{\circ} \mathrm{C}$. Furthermore, the same annealed sample shows a significant drop in the transmittance spectrum in the infrared region (1200-2500 nm), a clear evidence of a significant increase on its opacity and absorbing nature. Anyway, the semi-transparent character of the as-deposited sample was actually expected taking into account some recent studies in the group [27, 29]. The reflectance spectrum also reveals the signature of the onset of interband transition involving $d$-electrons in Ag [30], clearly visible for a wavelength value close to $320 \mathrm{~nm}$. The annealing-induced changes are actually extended over the entire spectrum, which can be attributed to the free electrons response. However, the reflectance is far below the unit for both as-deposited and annealed samples, which might result from two main reasons: (i) non-specular scattering and (ii) discontinuity of the metallic structure, i.e., its fractal geometry. Well defined shapes of Ag clusters would manifest themselves by their characteristic spectral features, for instance, spherical NPs (not too small) would show LSPR close to $400 \mathrm{~nm}[27,31]$, which would be splitted into two distinct resonances in nanorods. Fractal aggregates incorporate a 
plenitude of shapes and spatial scales and therefore their spectral response is very broad and almost featureless, as predicted theoretically and shown experimentally for specially prepared agglomerates of Ag NPs [18, 20,21].

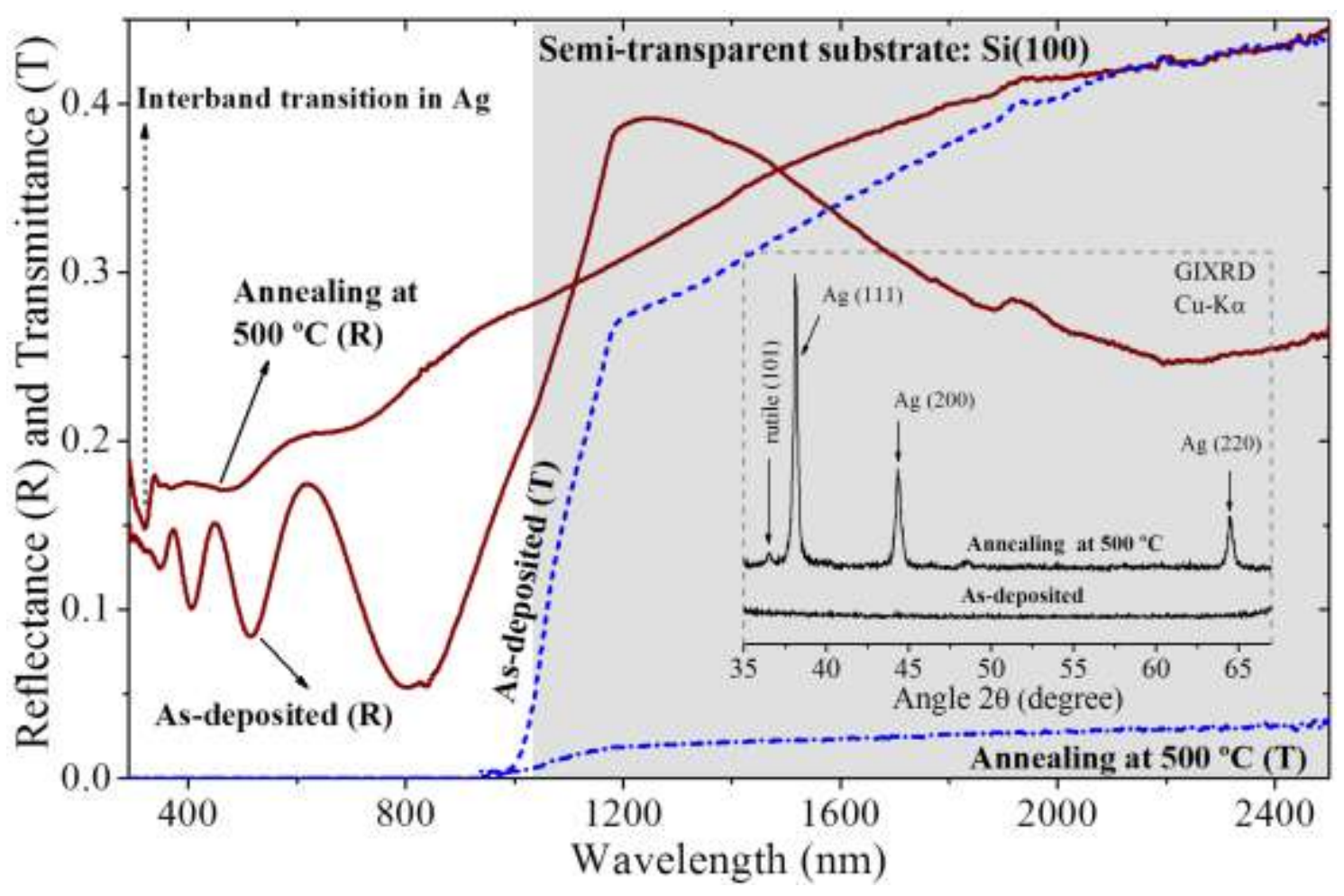

Figure 1. Reflectance and transmittance spectra of the Ag: $\mathrm{TiO}_{2}$ films, before and after annealing at $500{ }^{\circ} \mathrm{C}$. The inset shows the corresponding X-ray diffractograms of the samples.

The changes observed in the optical behaviour of the annealed sample are related with the crystallization and clustering of $\mathrm{Ag}$, as evidenced by the XRD diffractograms (see insert in Fig. 1). The XRD analysis of the films was performed in a Bruker D8 Discover, using copper radiation $(\mathrm{Cu}-\mathrm{K} \alpha)$ in grazing incidence mode $\left(\theta=2^{\circ}\right)$. The asdeposited sample is amorphous [27], but as soon as it is subjected to annealing treatment, the Ag diffusion throughout the host matrix occurs, and crystalline $\mathrm{Ag}$ 
domains are formed [27]. The Ag clusters crystallized in a face centered cubic (fcc) structure [COD card No. 1100136], as demonstrated by the particular diffraction patterns in Fig. 1. Furthermore, it seems that the matrix is poorly crystalline since the indexed rutile- $\mathrm{TiO}_{2}$ diffraction peak [COD card No. 9004141] is broad and revealed very low intensity, compared with those of crystalline Ag.

The surface morphology of the films was explored by Scanning Electron Microscopy (SEM), using a FEC-SEM Nova 200 NanoSEM scanning microscope. Figure 2 shows the results of such analysis, where one can easily notice the formation of not very common microstructures for this kind of films; namely, fractal aggregates of $\mathrm{Ag}$, dispersed over the surface of the thin film. The complex and branching dendrite-type objects are about $1 \mathrm{~mm}$ in lateral size, and only few hundred of nanometers in height (about $0.40 \mu \mathrm{m}$, as revealed by cross-sectional electron microscopy studies). Their form and low height-to-size ratio suggest a diffusion-limited growth process, which is governed by the 2D diffusion equation, with moving boundary conditions [32]. This aggregation phenomenon, commonly referred as diffusion-limited aggregation (DLA), is known to be far from equilibrium, and predicts that fractal geometries can emerge from homogeneous media, such as the case of the as-deposited sample (Ag embedded in $\mathrm{TiO}_{2}$ ). Under high temperature conditions, $\mathrm{Ag}$ atoms appear on the surface, owing to the segregation process, indicating a weak interaction with the matrix [33]. These adatoms, with high mobility, form a cluster at some singular point of the surface (a nucleation center), and certain area around this cluster becomes devastated because all of them stick to the cluster. However, Ag adatoms continue to appear at some distance from the cluster, moving towards it by means of a random walk and, eventually, stick to the growing aggregate irreversibly. This is the microscopic mechanism of the DLA fractal growth, which was modelled by T.A. Witten and L.M. Sander [34]. 


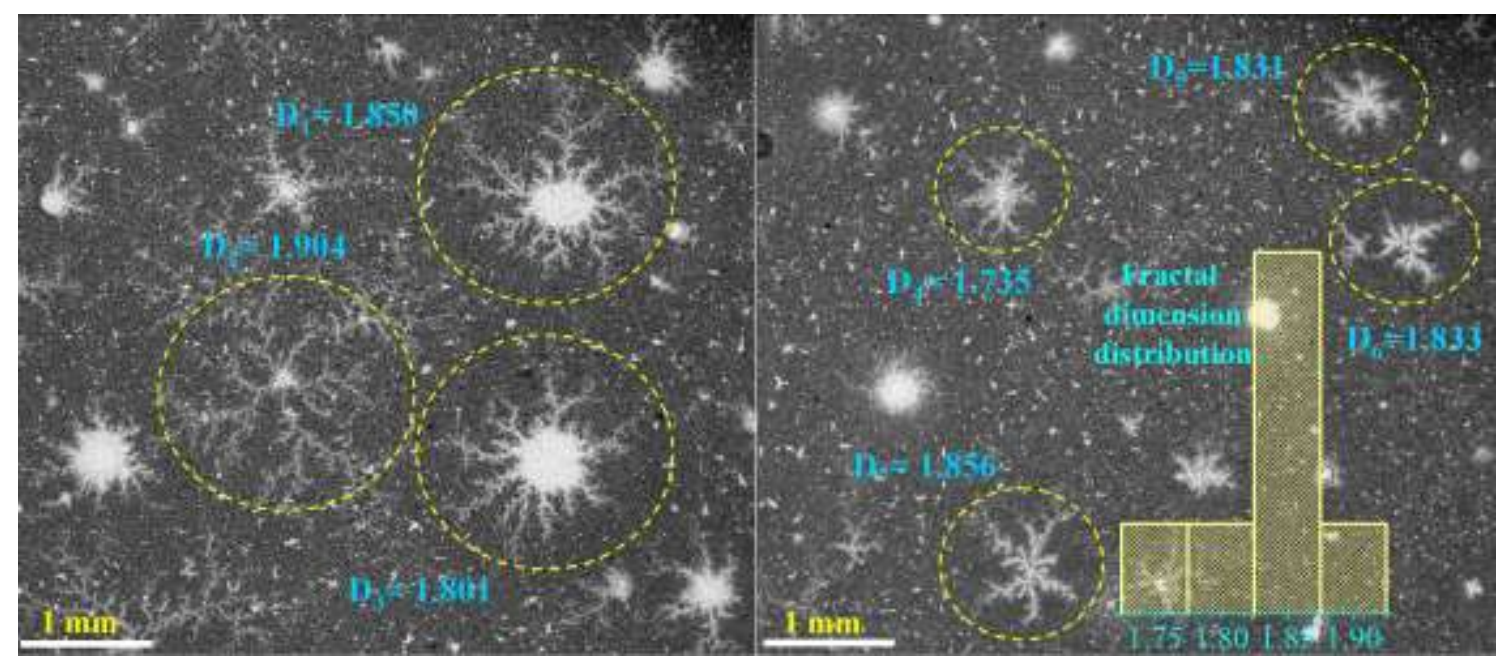

Figure 2. Overview of $\mathrm{Ag}$ fractal aggregates distributed over a $\mathrm{TiO}_{2}$ film. The fractal dimensions of selected fractals were calculated by applying the grid counting method to the digitalized images obtained by SEM.

Although the majority of the fractals observed in Fig. 2 have a dendrite-type structure, there are some noticeable differences in their geometries. In order to obtain quantitative data about the geometrical shapes, some fractals were chosen to be characterized in terms of fractal dimension $(D)$, using the digitalized images obtained by SEM. For the calculation of $D$ it was assumed that the fractal dimension of the projection of an aggregate onto a dimensional plane (2D) is the same as its original fractal dimension (3D) [32]. After application of basic image enhancement thresholding and binarization operators, the binary images were covered with different grids (with box lengths $\varepsilon$ ). The number of boxes, $N(\varepsilon)$, required to cover the structure of the fractal image was recorded for each $\varepsilon$. Then the fractal (Hausdorff) dimension was determined as $D=\lim _{\varepsilon \rightarrow 0}[-\log N(\varepsilon) / \log \varepsilon][35]$.

The results of the calculations are presented in Fig. 2 for the selected fractals, where a histogram with all obtained values of the fractal dimension is also shown. The highest 
frequency count is within the range 1.825 to 1.875 , which is in agreement with the values found in literature for DLA fractals, grown in a 2D Euclidean space [32].

Analysing the surface of the films in detail, a selected region near the branch of a fractal was successively zoomed-in, until individual clusters not attached to the structure were observed, Fig. 3.

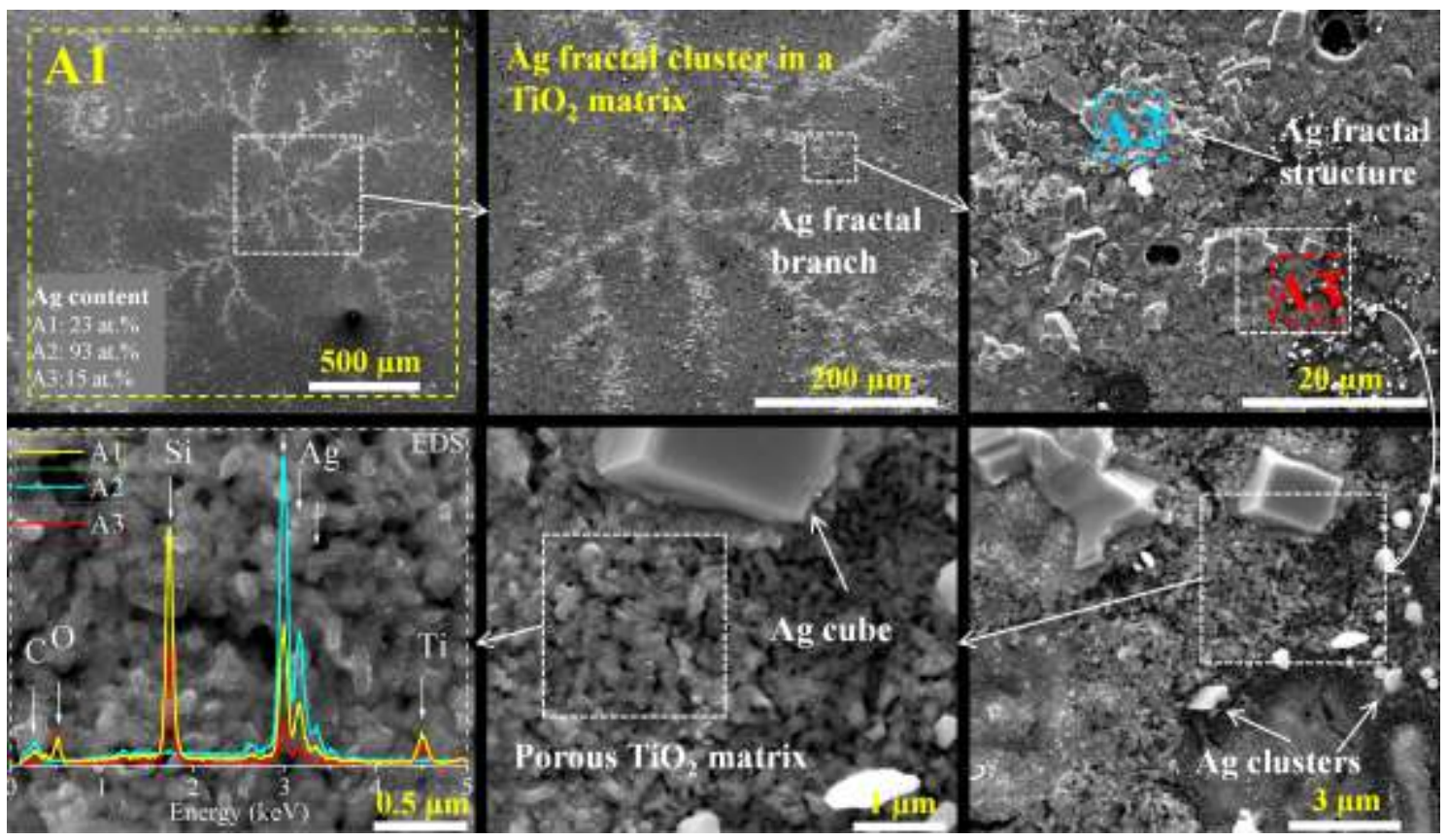

Figure 3. Microstructural details of the interface between the fractal structure and the surroundings. The EDS analysis was performed in the identified areas and the results are also embedded in the figure.

The existence of individual clusters in the vicinity of the fractal can be confirmed; some of them relatively small, which may correspond to the early stages of their formation, while others are relatively larger, with cubic shapes. This means that there are still some Ag clusters spread over the matrix, which are not attached to the body of the fractal. Therefore, the annealing stages might be optimized if one intends to maximize the formation of fractal structures, namely in terms of their size distribution and according 
to the specificities of the targeted applications. Furthermore, the oxidized state of the matrix is also an important parameter to take into account with respect to this process, since it may control the number of point defects (e.g. oxygen vacancies), which mediate the clusters' growth on the surface of $\mathrm{TiO}_{2}$ [36]. As claimed by J. Hansen et al. [33], the interaction of $\mathrm{Ag}$ clusters with $\mathrm{TiO}_{2}$ is stronger as the latter is more oxidized. However, in this work, Ag atoms migrated significantly to the surface to form the fractal clusters, which was probably the result of the fairly low chemical interaction of Ag clusters to the roughly stoichiometric matrix.

Additional studies were performed using Energy Dispersive X-ray Spectroscopy (EDS). The EDS analysis of the films confirmed, as expected, that the main body of the fractal is composed of $\mathrm{Ag}$, surrounded by the $\mathrm{TiO}_{2}$ matrix. The selected area $\mathrm{A} 1$ (Fig. 3) was analysed using EDS, giving an atomic concentration of $\mathrm{Ag}$ of about 23 at.\% (in agreement with RBS analysis), confirming also the roughly stoichiometric $\mathrm{TiO}_{2}$. When the electron beam is focused in a smaller area, which only includes a part of the branch of the fractal, the EDS signal of Ag is clearly enhanced, corresponding to the atomic concentration as high as 93 at.\%; a clear evidence of the presence of this peculiar metallic microstructure. In this case, the substrate ( $\mathrm{Si})$ signal clearly vanishes, which is certainly the result of the fact that the scanning electrons cannot pass through Ag (lower mean free path in comparison with $\mathrm{TiO}_{2}$ ) [37]. Finally, when the electron beam is focused in the adjacent area A3, containing both Ag clusters and the matrix (not representative of the whole film), but still with the same size as A2, the Si EDS peak appears again, giving further evidence of the electronic transparency of $\mathrm{TiO}_{2}$ formed under the present experimental conditions.

Finally, another major finding in this work is related with the particular microstructure of the matrix, which is developed as a consequence of the fractal formation (a result of 
the annealing process at moderate temperature $-500{ }^{\circ} \mathrm{C}$ ). SEM analysis of fractalformed samples suggests that the oxide matrix becomes very porous, especially evident in the last two micrographs of Fig. 3. From this perspective, and beyond the particular change in the optical response of the films, the development of a porous matrix can also enhance the performance of some devices that might be used for the detection of molecules in SERS [21, 38], or in biosensing-driven applications if combined with luminescent QDs [11]. Porous matrixes can facilitate the interaction between the analyte molecules and the plasmonic structure, providing efficient binding sites for the capture of molecules [39]. As a matter of fact, the number of contacts between Ag clusters and analytes is expected to increase significantly since the surface area available for the target molecules is much higher and their diffusion throughout the porous matrix is enhanced [40, 41]. However, in any case it is also important to take into account the oxide state of the surface of Ag clusters, since the chemical binding to analytes might be affected by it. In a previous work, X-Ray Photoelectron Spectroscopy (XPS) analysis was performed in $\mathrm{Ag}: \mathrm{TiO}_{2}$ films, and from those results it was possible to claim that $\mathrm{Ag}$ surface is most probably oxidized (see ref. [27]). This conclusion is based on the negative shift $(\Delta \mathrm{E}=-0.37 \mathrm{eV})$ observed for the $\mathrm{Ag}-3 \mathrm{~d}_{5 / 2}$ peak, in comparison to metallic silver $(368.27 \mathrm{eV})$ [27]. Therefore, assuming that the Ag fractal structures are oxidized, the sample might need plasma activation (e.g. using Ar or $\mathrm{H}_{2}$ plasma) in order to turn it more reactive to the targeted molecules.

In conclusion, it was shown that it is possible to produce Ag fractal structures on top of a $\mathrm{TiO}_{2}$ porous matrix, but only after subjecting the sputtered $\mathrm{Ag}: \mathrm{TiO}_{2}$ thin films to thermal annealing at $500{ }^{\circ} \mathrm{C}$. The formation of metallic DLA-type fractal structures opens the possibility of using them in applications where the enhancement of local electromagnetic field are required, such as SERS [20, 21, 38]. Furthermore, the 
interaction between the porous metal oxide matrix and analyte molecules, through either chemical reaction or physical adsorption, can induce a significant change in the optical properties of the fractal structures. This may also be considered for optical SPR-sensing in the detection of small concentrations of (bio-) molecules [25, 42].

\section{Acknowledgements}

This research was sponsored by FEDER funds through the COMPETE program (Programa Operacional Factores de Competitividade) and by FCT (Fundação para a

Ciência e a Tecnologia), under the projects UID/FIS/04650/2013 and

PEstOE/MAT/UI0013/2014. A. Ferreira and C. Lopes acknowledge also FCT for grants SFRH/BPD/102402/2014 and SFRH/BD/103373/2014.

References

[1] M. Torrell, R. Kabir, L. Cunha, M.I. Vasilevskiy, F. Vaz, A. Cavaleiro, E. Alves, N.P. Barradas, Journal of Applied Physics 109/7 (2011) 074310.

[2] N.M. Figueiredo, T. Kubart, J.A. Sanchez-García, R. Escobar Galindo, A. ClimentFont, A. Cavaleiro, Journal of Applied Physics 115/6 (2014) 063512.

[3] J. Borges, M. Buljan, J. Sancho-Parramon, I. Bogdanovic-Radovic, Z. Siketic, T. Scherer, C. Kübel, S. Bernstorff, A. Cavaleiro, F. Vaz, A.G. Rolo, Journal of Nanoparticle Research 16/12 (2014) 2790.

[4] J. Preclíková, F. Trojánek, P. Němec, P. Malý, physica status solidi (c) 5/11 (2008) 3496.

[5] L. Polavarapu, J. Perez-Juste, Q.-H. Xu, L.M. Liz-Marzan, Journal of Materials Chemistry C 2/36 (2014) 7460.

[6] D. O'Connor, A.V. Zayats, Nat Nano 5/7 (2010) 482.

[7] X. Gu, T. Qiu, W. Zhang, P.K. Chu, Nanoscale Res. Lett. 6/1 (2011) 1.

[8] H. Choi, S.-J. Ko, Y. Choi, P. Joo, T. Kim, B.R. Lee, J.-W. Jung, H.J. Choi, M. Cha, J.R. Jeong, I.-W. Hwang, M.H. Song, B.-S. Kim, J.Y. Kim, Nat Photon 7/9 (2013) 732.

[9] C.L. Nathan, N. Prashant, M.M. Kevin, J.N. David, O. Sang-Hyun, Reports on Progress in Physics 75/3 (2012) 036501.

[10] P. Törmä, W.L. Barnes, Reports on Progress in Physics 78/1 (2015) 013901.

[11] I. Moura, M.F. Cerqueira, D. Melnikau, D. Savateeva, Y. Rakovich, J. Borges, F. Vaz, M. Vasilevskiy, Journal of Physics: Conference Series 605/1 (2015) 012025.

[12] M. Logar, B. Jančar, S. Šturm, D. Suvorov, Langmuir 26/14 (2010) 12215.

[13] P. Christopher, D.B. Ingram, S. Linic, The Journal of Physical Chemistry C 114/19 (2010) 9173.

[14] S. Linic, U. Aslam, C. Boerigter, M. Morabito, Nat Mater 14/6 (2015) 567.

[15] L. Enzhou, K. Limin, Y. Yuhao, S. Tao, H. Xiaoyun, Z. Changjun, L. Hanchen, W.

Qiuping, L. Xinghua, F. Jun, Nanotechnology 25/16 (2014) 165401.

[16] J. Toudert, H. Fernandez, D. Babonneau, S. Camelio, T. Girardeau, J. Solis, Nanotechnology 20/47 (2009) 475705. 
[17] C.G. Khoury, T. Vo-Dinh, The Journal of Physical Chemistry C 112/48 (2008) 18849.

[18] V.A. Markel, V.M. Shalaev, E.B. Stechel, W. Kim, R.L. Armstrong, Physical Review B 53/5 (1996) 2425.

[19] A.V. Zenkevich, M.A. Pushkin, V.N. Tronin, V.I. Troyan, V.N. Nevolin, G.A. Maximov, D.O. Filatov, E. Lægsgaard, Physical Review B 65/7 (2002) 073406.

[20] A.K. Buin, P.F. de Châtel, H. Nakotte, V.P. Drachev, V.M. Shalaev, Physical Review B 73/3 (2006) 035438.

[21] A. Biswas, H. Eilers, F. Hidden, O.C. Aktas, C.V.S. Kiran, Applied Physics Letters 88/1 (2006) 013103.

[22] A. Biswas, I.S. Bayer, D.H. Dahanayaka, L.A. Bumm, Z. Li, F. Watanabe, R. Sharma, Y. Xu, A.S. Biris, M.G. Norton, E. Suhir, Nanotechnology 20/32 (2009) 325705.

[23] J. Borges, N.P. Barradas, E. Alves, M.F. Beaufort, D. Eyidi, F. Vaz, L. Marques, Journal of Physics D: Applied Physics 46/1 (2013) 015305.

[24] R.M.S. Pereira, J. Borges, F.C.R. Peres, P.A.S. Pereira, G.V. Smirnov, F. Vaz, A. Cavaleiro, M.I. Vasilevskiy, Journal of Nanophotonics 9/1 (2015) 09736.

[25] P. Zhang, S. Wang, ChemPhysChem 15/8 (2014) 1550.

[26] J. Borges, T. Kubart, S. Kumar, K. Leifer, M.S. Rodrigues, N. Duarte, B. Martins, J.P. Dias, A. Cavaleiro, F. Vaz, Thin Solid Films 580/0 (2015) 77.

[27] J. Borges, M.S. Rodrigues, C. Lopes, D. Costa, F.M. Couto, T. Kubart, B. Martins, N. Duarte, J.P. Dias, A. Cavaleiro, T. Polcar, F. Macedo, F. Vaz, Applied Surface Science 358, Part B (2015) 595.

[28] C. Pascual-Izarra, M.A. Reis, N.P. Barradas, Nuclear Instruments and Methods in Physics Research Section B: Beam Interactions with Materials and Atoms 249/1-2 (2006) 780. [29] J. Borges, M.S. Rodrigues, T. Kubart, S. Kumar, K. Leifer, M. Evaristo, A. Cavaleiro, M. Apreutesei, R.M.S. Pereira, M.I. Vasilevskiy, T. Polcar, F. Vaz, Thin Solid Films 596 (2015) 8 .

[30] J.A. Scholl, A.L. Koh, J.A. Dionne, Nature 483/7390 (2012) 421.

[31] J. Chen, S. Shi, R. Su, W. Qi, R. Huang, M. Wang, L. Wang, Z. He, Sensors (Basel, Switzerland) 15/6 (2015) 12205.

[32] T. Vicsek, Fractal Growth Phenomena, World Scientific, 1992.

[33] J.Ø. Hansen, E. Lira, P. Galliker, J.-G. Wang, P.T. Sprunger, Z. Li, E. Lægsgaard, S. Wendt, B. Hammer, F. Besenbacher, The Journal of Physical Chemistry C 114/40 (2010) 16964.

[34] T.A. Witten, L.M. Sander, Physical Review Letters 47/19 (1981) 1400.

[35] D. Melnikau, D. Savateeva, V. Lesnyak, N. Gaponik, Y.N. Fernandez, M.I. Vasilevskiy, M.F. Costa, K.E. Mochalov, V. Oleinikov, Y.P. Rakovich, Nanoscale 5/19 (2013) 9317.

[36] Z.D. Davis, B.J. Tatarchuk, Applied Surface Science 353 (2015) 679.

[37] R.F. Egerton, Electron Energy-Loss Spectroscopy in the Electron Microscope, Springer US, 2011.

[38] V. Iancu, L. Baia, N. Tarcea, J. Popp, M. Baia, Journal of Molecular Structure 1073 (2014) 51.

[39] S. Fateixa, H.I.S. Nogueira, T. Trindade, Physical Chemistry Chemical Physics 17/33 (2015) 21046.

[40] M.-H. Seo, M. Yuasa, T. Kida, J.-S. Huh, K. Shimanoe, N. Yamazoe, Sensors and Actuators B: Chemical 137/2 (2009) 513.

[41] X. Zhang, Y. Zheng, X. Liu, W. Lu, J. Dai, D.Y. Lei, D.R. MacFarlane, Advanced Materials 27/6 (2015) 1090.

[42] K.M. Byun, N.-H. Kim, J.W. Leem, J.S. Yu, Applied Physics B 107/3 (2012) 803. 